\title{
Personalised nutrition advice delivered online or face-to-face is more effective at motivating healthier dietary choices than generalised advice in Kuwait
}

\author{
Balqees Alawadhi ${ }^{1}$, Rosalind Fallaize ${ }^{1}$, Rodrigo Zenun ${ }^{2}$, Faustina Hwang ${ }^{2}$ and Julie Lovegrove ${ }^{1}$ \\ ${ }^{1}$ Hugh Sinclair Unit of Human Nutrition and Institute for Cardiovascular and Metabolic Research, University of \\ Reading, Reading, United Kingdom and \\ ${ }^{2}$ Biomedical Engineering, School of Biological Sciences, University of Reading, Reading, United Kingdom
}

\section{Abstract}

Introduction: The adoption of poor dietary and lifestyle habits have been associated with the development of non-communicable disease. The majority of strategies implemented to enhance dietary quality of individuals follow a "one size fits all" standardised approach. Results of recent trials have suggested that Personalised Nutrition (PN), tailored to individual requirements, is able to improve dietary intakes, yet limited focus has been given to the effectiveness of face-to-face compared with online methods. The aim of the EatWellQ8 randomised control trial (RCT) was to assess the impact of web-based PN advice, face-to-face PN advice and standardised advice, on adherence to healthy eating in Kuwait.

Materials and Methods: Free living adults aged 21-65 years, were recruited for the 12-week study and randomised to; face-to-face PN, web-based PN or generalised (control) advise groups. Dietary intake and self-reported anthropometric measurements were assessed at baseline, 6 and 12 weeks. A validated food frequency questionnaire (FFQ) modified from the EPIC FFQ was used to assess food and nutrient intake. Diet quality was assessed by a 10-component modified Alternative Healthy Eating Index (m-AHEI) which was used to generate the PN advice. At 0 and 12-weeks post FFQ completion, participants randomised to the PN intervention groups were presented with 3 tailored dietary messages based on the m-AHEI components that received the lowest scores.

Results: 320 participants completed the trial. Due to over/underreporting, 100 were included in the analysis (71\% female, $29 \%$ male) with a mean age of 38.6 years (SD 14.3), and body mass index (BMI) of $25.1 \mathrm{~kg} / \mathrm{m}^{2}$ (SD 4.2). After 12-weeks intervention, m-AHEI scores increased significantly in both PN intervention groups (face-to-face PN 19\%, web-based $12 \%)$ compared to controls $(4 \%)(P<$ $0.01)$ and significantly higher intakes of vegetables and fruits, and lower intakes of sugars compared with controls $(\mathrm{P}<0.05)$. The $\mathrm{PN}$ intervention groups also significantly increased their intakes of omega 3 fatty acids and total folate compared with the control group $(\mathrm{P}<0.05)$. The Face-to-face PN group significantly reduced weight $(-1.9 \mathrm{~kg})$ and BMI $\left(-0.5 \mathrm{~kg} / \mathrm{m}^{2}\right)$ compared to web-based PN and control groups $(\mathrm{P}<0.01)$.

Discussion: In adults living in Kuwait, PN advice, delivered face-to-face or online, was more effective at improving dietary quality than population-based advice. Face-to-face PN was found to be more effective at inducing weight-loss in adults compared to web-based $\mathrm{PN}$ and population-based advice.

\section{Conflict of Interest}

There is no conflict of interest 